\title{
Tratamento cirúrgico de fratura do complexo zigomático orbitário: Relato de caso
}

\author{
Surgical treatment of orbital zygomatic complex fracture: Case report \\ Tratamiento quirúrgico de la fractura del complejo cigomático orbitario: Reporte de caso
}

Recebido: 01/07/2021 | Revisado: 09/07/2021 | Aceito: 13/07/2021 | Publicado: 23/07/2021

\author{
Eugênia Leal de Figueiredo \\ ORCID: https://orcid.org/0000-0001-5210-1389 \\ Universidade Federal de Pernambuco, Brasil \\ E-mail: eugeniafigueiredo@ hotmail.com \\ Lucas Peron Cavalcanti \\ ORCID: https://orcid.org/0000-0001-6069-3820 \\ Centro Universitário Brasileiro, Brasil \\ E-mail: lucas-peron@hotmail.com \\ Jeanine Angelo da Silva \\ ORCID: https://orcid.org/0000-0001-9988-3904 \\ Centro Universitário Brasileiro, Brasil \\ E-mail: jeanineangela1995@gmail.com \\ Davi Felipe Neves Costa \\ ORCID: https://orcid.org/0000-0003-3458-9696 \\ Universidade Federal da Paraíba, Brasil \\ E-mail: Davifelipecosta@gmail.com \\ Jair Carneiro Leão \\ ORCID: https://orcid.org/0000-0001-8303-2291 \\ Universidade Federal de Pernambuco, Brasil \\ E-mail: jair.leao@ufpe.br \\ Alessandra de Albuquerque Tavares Carvalho \\ ORCID: https://orcid.org/0000-0003-0390-3611 \\ Universidade Federal de Pernambuco, Brasil \\ E-mail: alessandra.tcarvalho@ufpe.br
}

\begin{abstract}
Resumo
Os casos de fratura do complexo zigomático orbitário têm altos índices, logo, é necessário o conhecimento de seus sinais e sintomas, pois possibilita um diagnóstico precoce e um melhor prognóstico. Este trabalho é um relato de um caso clínico de fratura do complexo zigomático orbitário, onde foram descritos os sinais e sintomas clínicos observando disfunções estéticas e funcionais a fim de fornecer um melhor diagnóstico e planejamento cirúrgico e de avaliar as técnicas empregadas, mostrando a importância do cirurgião bucomaxilofacial no tratamento desses tipos de fraturas. Paciente do sexo masculino procurou o serviço de Cirurgia e Traumatologia Buco-Maxilo-Facial, vítima de acidente automobilístico. No exame físico foram identificados: ptose palpebral, equimose periorbitária, hiposfagma, diplopia e oftalmoplegia no olho direito, enoftalmia, perda de projeção ântero-posterior do zigomático, edema difuso em terço médio de face, alargamento de terço médio de face e limitação de abertura bucal. Foi solicitado o exame tomográfico o qual confirmou as fraturas no complexo zigomático e a presença de solução de continuidade compatível com fratura blow-out, seguindo para procedimento cirúrgico. Paciente foi submetido a 3 acessos cirúrgicos, 3 pontos de fixação interna (com placas e parafusos do sistema de fixação de 1,5mm, 2,0mm) e colocação de tela orbitária para reconstrução do assoalho de órbita. Obteve-se sucesso no tratamento, mostrando que o procedimento cirúrgico é seguro e eficaz, resultando em ganho estético e funcional.
\end{abstract}

Palavras-chave: Fraturas zigomáticas; Fixação de fratura; Fraturas cominutivas.

\begin{abstract}
The cases of fracture of the orbital zygomatic complex have high rates, therefore, knowledge of its signs and symptoms is necessary, as it allows for an early diagnosis and a better prognosis. The present work is a clinical case report of a fracture in the orbital zygomatic complex, where the clinical signs and symptoms were described observing aesthetic and functional dysfunctions in order to provide a better diagnosis and surgical planning and to evaluate the techniques used, showing the importance of maxillofacial surgeon in the treatment of these types of fractures. Male patient sought the Maxillofacial Surgery and Traumatology service, victim of a car accident. On physical examination, were identified: palpebral ptosis, periorbital ecchymosis, hyposphagma, diplopia and ophthalmoplegia in the right eye, enophthalmia, loss of anteroposterior projection of the zygomaticus, diffuse edema in the middle third of the face, enlargement of the middle third of the face and mouth opening limitation. A tomographic exam was requested, which confirmed the fractures in the zygomatic complex and the presence of continuity solution compatible with a blow-out fracture, followed by the surgical procedure. The patient underwent 3 surgical approaches, 3 internal
\end{abstract}


fixation points (with $1.5 \mathrm{~mm}, 2.0 \mathrm{~mm}$ fixation system plates and screws) and placement of an orbital mesh for reconstruction of the orbital floor. The treatment was successful, showing that the surgical procedure is safe and effective, resulting in aesthetic and functional gain.

Keywords: Zygomatic fractures; Fracture fixation; Comminuted fractures.

\begin{abstract}
Resumen
Los casos de fractura del complejo cigomático orbitario tienen altas tasas, por lo que es necesario el conocimiento de sus signos y síntomas, ya que permite un diagnóstico precoz y un mejor pronóstico. Este trabajo es el reporte de un caso clínico de fractura del complejo cigomático orbitario, donde se describieron los signos y síntomas clínicos observando disfunciones estéticas y funcionales con el fin de brindar un mejor diagnóstico y planificación quirúrgica y evaluar las técnicas utilizadas, mostrando la importancia del cirujano maxilofacial en el tratamiento de este tipo de fracturas. Paciente de sexo masculino que acude al servicio de Cirugía y Traumatología Maxilofacial, víctima de un accidente de tráfico. Al examen físico se identificaron: ptosis palpebral, equimosis periorbitaria, hiposfagma, diplopía y oftalmoplejía en el ojo derecho, enoftalmia, pérdida de proyección anteroposterior del cigomático, edema difuso en el tercio medio de la cara, agrandamiento del tercio medio de la cara y limitación de apertura oral. Se solicitó un examen tomográfico, que confirmó las fracturas en el complejo cigomático y la presencia de solución de continuidad compatible con una fractura por estallido, seguido del procedimiento quirúrgico. El paciente fue sometido a 3 abordajes quirúrgicos, 3 puntos de fijación internos (con placas y tornillos del sistema de fijación de 1,5 mm, 2,0 mm) y colocación de una malla orbitaria para la reconstrucción del suelo orbitario. El tratamiento fue exitoso, demostrando que el procedimiento quirúrgico es seguro y efectivo, lo que resulta en una ganancia estética y funcional.
\end{abstract}

Palabras clave: Fracturas cigomáticas; Fijación de fracturas; Fracturas conminutas.

\title{
1. Introdução
}

Segundo Freire (2001), o conceito de trauma é visto como um grupo das perturbações provocadas abruptamente por um agente físico de etiologia, índole e aumento diversos, sendo capaz de estar localizado em várias partes do corpo e estão entre as primeiras causas de óbito e morbidade na humanidade, de acordo com a Organização Mundial de Saúde. Entre os traumas mais comuns estão as fraturas faciais que apresentam uma ocorrência instável segundo a idade, gênero, área geográfica, dados culturais, classe social, intervenção climática, consumo de álcool e drogas ilícitas, violação das leis de trânsito, acidentes motociclístico, violência doméstica, anomalias ósseas e fatores etiológicos (Allaredy et al., 2011; Rampa et al., 2019).

A análise e a intervenção das fraturas do complexo zigomático (CZO) requerem uma equipe multidisciplinar, abrangendo essencialmente as especialidades de trauma, oftalmologia, cirurgia plástica, neurocirurgia e cirurgia bucomaxilofacial. Considerando o elevado índice e a predominância dos traumatismos faciais, o atendimento primário do paciente com o bucomaxilofacial exige a compreensão dos princípios gerais, é fundamental conhecer os tipos de lesões que afetam a face, para que possam cooperar nos primeiros socorros, como, também dos princípios gerais do paciente politraumatizado, haja visto que essas lesões comumente se encontram associadas a outras, de diversas partes do corpo. Portanto, o paciente deve ser atendido, avaliado e tratado de forma sistemática e interdisciplinar, de acordo com o que se preconiza no protocolo do ATLS (Advanced Trauma Life Support) (Oliveira, 2011; Souza et al, 2010; Martins et al, 2013).

Estas fraturas faciais vêm sendo mais comum a cada dia, principalmente em cidades grandes e em locais com intensa movimentação de indivíduos e de automóveis (Batstone et al., 2007; Montovani et al., 2006). Homens com idade superior a 25 anos são os mais propensos à essas fraturas, isto normalmente ocorre devido à falta do uso de capacetes quando estão em motocicletas e ao desacato às normas de velocidade (Baird et al., 2004; Perry et al., 2008).

A aproximação do arcabouço facial com a superfície intracraniana e pela razão da face ser uma área bem evidente no corpo humano faz com que fraturas neste local sejam consideradas fraturas com potencial de alterações estéticas, que pode dar origem a sequelas definitivas, e até mesmo levando o paciente à óbito. É de suma importância reconhecer as causas, o intervalo de idade, gênero mais afetado e pontos anatômicos mais acometidos para que se desenvolva métodos de prevenção e evitar que o número de fraturas faciais continue a aumentar (Erdmann et al., 2008). 
Entre as lesões faciais, as fraturas do (CZO) são capazes de comprometer a funcionalidade e a estética facial, pois estão intimamente conectadas com outras estruturas nobres da face, como por exemplo, a órbita, o que torna o tratamento de extrema importância (Peretti et al., 2017). Os sinais e sintomas mais identificados nessas fraturas são: hemossinus e epistaxe, diplopia, edema, enoftalmo, distopia, hematoma, equimose subconjuntival, limitação da motricidade ocular (oftalmoplegia), assimetria facial (por afundamento da região zigomática) e ectrópio (Souza et al., 2009; Peretti et al., 2017). O diagnóstico e a intervenção antecipada das fraturas do CZO possibilitam um melhor resultado funcional e estético.

O diagnóstico dessas fraturas é efetivado por meio do exame clínico e exame de imagem. No exame clínico deve-se observar o paciente, as lacerações, abrasões e áreas equimóticas. É recomendado analisar evidências de epistasia e extravasamento de líquido cefalorraquidiano, e também avaliar a assimetria nasal e telecanto traumático. No exame intra-oral deve-se apurar a presença de dentes fraturados, equimose vestibular e palatina, edema, diastemas e má-oclusão. A palpação deve ser realizada rigorosamente, buscando por sinais de crepitações e mobilidade em todos os ossos da face, observando a maxila como um elemento separado ao mesmo temo que palpa a fronte procurando por sinais de afundamento ou deslocamento da mesma. O diagnóstico de imagem pode ser realizado pela tomografia computadorizada (TC), visto que hoje em dia, elas oferecem a melhor imagem e é mais confiável em distinguir as prováveis fraturas. Também é possível que as TCs apontem as diferenças do hematoma ou edema nos tecidos moles, por isso se torna um aparelho valioso na comprovação do diagnóstico (Miloro et al., 2004).

Contudo, lesões não diagnosticadas ou traumas com risco à vida, podem postergar o tratamento (Lu et al., 2012). As intervenções das fraturas faciais vão depender de várias condições e é mais prudente tratar assim que possível, uma vez que, quanto mais se demorar para realizar o tratamento, as chances de ocorrer sequelas aumentam, e o reposicionamento anatômico das estruturas ósseas fraturadas se torna mais difícil (Freitas, 2006).

Knight e North (1961), através de radiografias, classificou as fraturas do CZO em seis grupos, são eles: Grupo 1 Fratura sem deslocamento significativo. Grupo 2 Fratura de arco zigomático. Grupo 3 Fratura de corpo zigomático sem rotação. Grupo 4 Fratura de corpo zigomático com rotação medial. Grupo 5 Fratura de corpo zigomático com rotação lateral. Grupo 6 Fratura complexa (Prado et al, 2011).

Existem ainda fraturas que acometem particularmente o assoalho e/ou a parede medial orbitária, essas fraturas são denominadas de blow-out e frequentemente afetam a porção localizada medialmente ao canal infra-orbitário no assoalho e a lâmina papirácea do etmóide na parede medial (Burm et al, 1999).

Observando a grande quantidade de casos de fratura do complexo zigomático e o seu envolvimento com estruturas nobres da face, este estudo se faz necessário, pois o conhecimento dos seus sinais e sintomas possibilitam um diagnóstico precoce e elaboração de um planejamento cirúrgico ideal. A escolha correta do tratamento a ser empregado possibilita ao cirurgião bucomaxilofacial um melhor prognóstico nos procedimentos realizados, com a melhora das implicações estéticas e funcionais, as quais podem ocasionar sequelas devastadoras e até permanentes ao paciente.

Este trabalho tem como objetivo relatar um caso clínico de fratura do complexo zigomático orbitário, avaliando as disfunções estético e funcionais, a melhor forma de tratamento e a importância do cirurgião bucomaxilofacial no diagnóstico e tratamento destes tipos de fraturas.

\section{Metodologia}

Este estudo qualitativo, trata-se de um relato de caso que foi realizado por coleta direta dos dados do paciente por meio do acesso aos prontuários e exames. Os dados foram coletados após a realização da anamnese, exame físico e radiográfico, tendo o diagnóstico de fratura do complexo zigomático e fratura blow-out. Assim, foram analisados os sinais e sintomas, tratamento cirúrgico aberto e pós operatório do paciente. Buscou-se por estudos publicados entre o período de 1999 e 
2019, nos sites do Scientific Eletronic Library Online (SCIELO) e Medical Literature Analysis and Retrival System Online (MEDLINE), fazendo um cruzamento entre os descritores "Fraturas zigomáticas", "Fixação de fratura", "Fraturas cominutivas". Os artigos foram pesquisados nos idiomas português e inglês, selecionando os que contribuíram para a análise deste trabalho.

\section{Relato de Caso}

Paciente D.J.S, sexo masculino, leucoderma, 22 anos, procurou o serviço de Cirurgia e Traumatologia Buco-MaxiloFacial, vítima de acidente automobilístico. Ao exame físico (Figura 1-A), apresentava ptose palpebral, equimose periorbitária, hiposfagma, diplopia e oftalmoplegia no olho direito (Figura 1-B), enoftalmia, perda de projeção ântero-posterior do zigomático, edema difuso em terço médio de face, alargamento de terço médio de face e limitação de abertura bucal (Figura 1C).

Figura 1-A: Aspecto clinico pré-operatório. Figura 1-B: Oftalmoplegia no olho direito Figura 1-C: Fotografia pré-operatória demonstrando a limitação de abertura de bucal de $20 \mathrm{~mm}$.

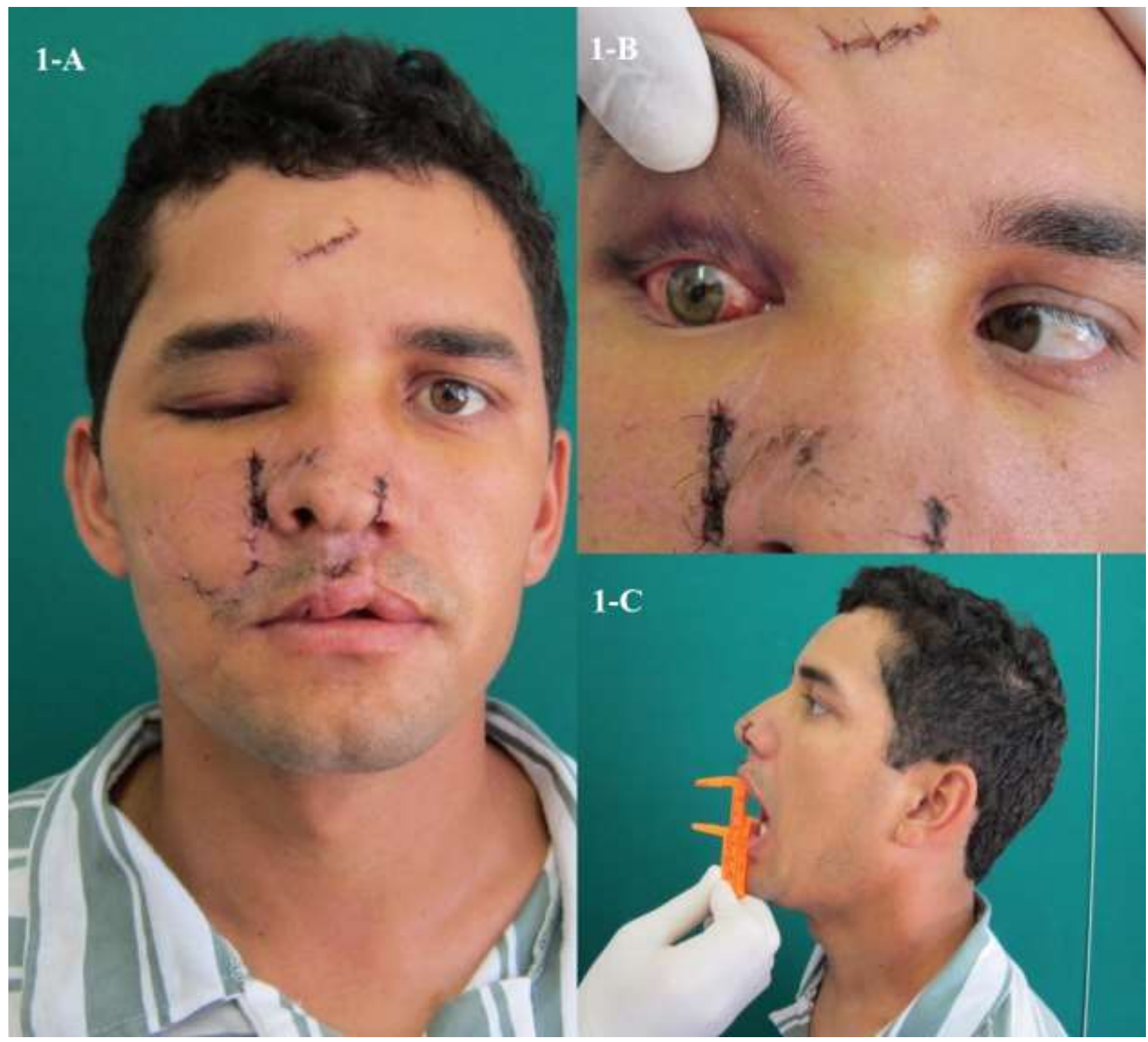

Fonte: Autores. 
Foi solicitado exame tomográfico (Figura 2-A) onde foi observado a presença de solução de continuidade compatível com fratura blow-out nos cortes coronal (Figura 2-B) e axial (Figura 2-C), e fraturas no complexo zigomático do lado direito. Com exame de imagem complementando o exame físico, foi observado todas as fraturas que acometeram à vítima, confirmando a hipótese de fratura blow-out e do complexo zigomático, estabelecendo assim o planejamento cirúrgico realizar três acessos cirúrgicos e três pontos de fixação interna e utilização de telas e placas de titânio.

Figura 2-A: Tomografia computadorizada pré-operatória. Figura 2-B: Corte coronal da tomografia computadorizada. Figura 2-C: Corte axial da tomografia computadorizada.

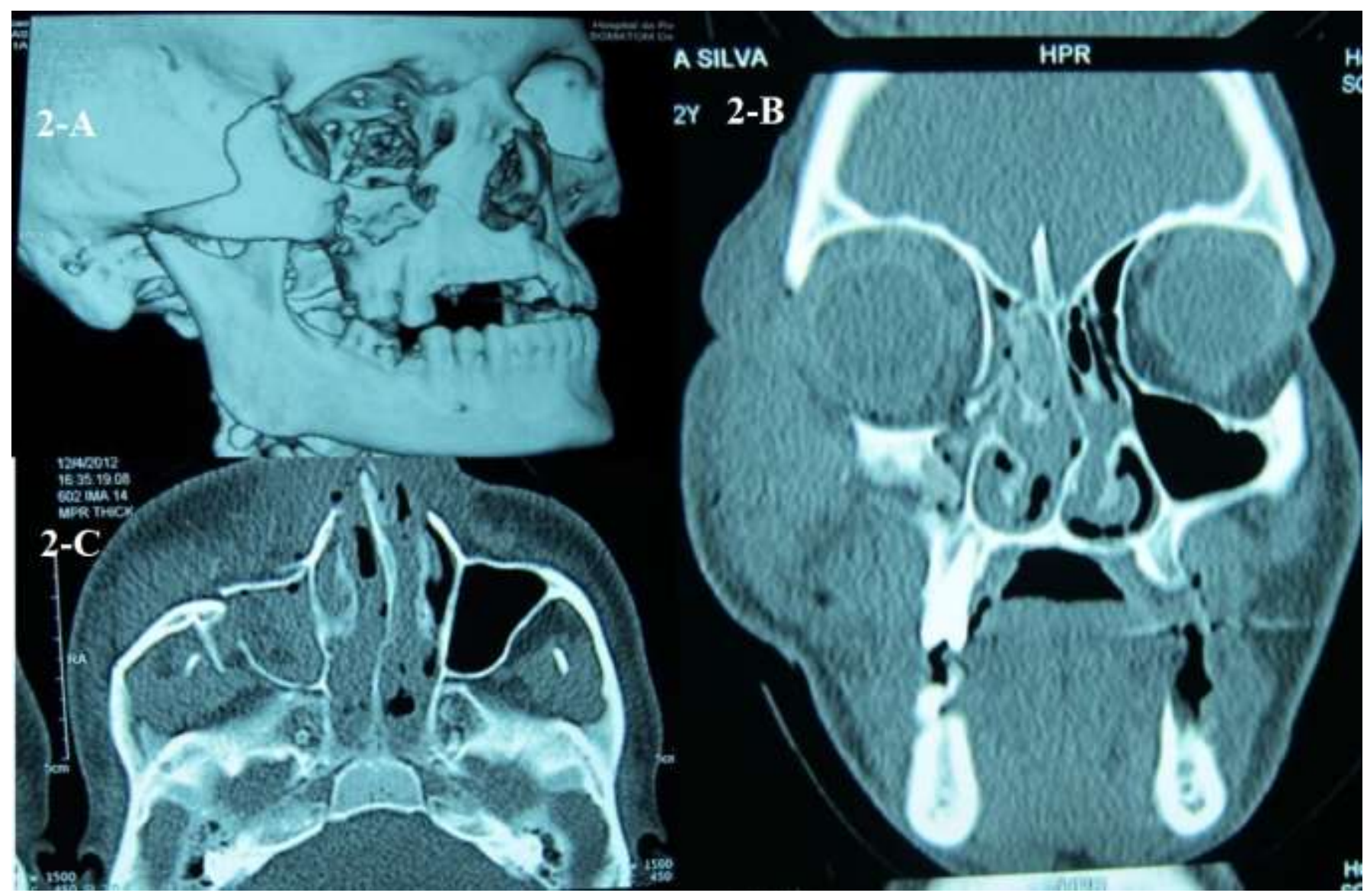

Fonte: Autores.

Após 7 dias o paciente foi submetido ao procedimento cirúrgico sob anestesia geral. Pelo padrão de fratura, foi colocada uma tela orbitária para reconstrução do assoalho de órbita. Foi feito o acesso supra-orbitário para instalação de 1 placa semilunar $2.0 \mathrm{~mm}$ na sutura fronto zigomática (Figura 3-A), acesso subtarsal para instalação de 1 placa $1.5 \mathrm{~mm} \mathrm{em}$ margem infra orbitária direita (Figura 3-B) e colocação de uma tela $1.5 \mathrm{~mm}$ no assoalho de órbita, acesso vestibular maxilar direita para colocação de uma placa em "L" no pilar zigomático (Figura 3-C). Na cirurgia foi realizado o teste de ducção forçada (Figura 3-D) antes e após a realização de redução e fixação de fratura blow-out e das fraturas no complexo zigomático. 
Figura 3-A: Acesso supra-orbitário com a placa semilunar na sutura Frontozigomática. Figura 3-B: Colocação de placa 1.5mm na margem infraorbitária Figura 3-C: Colocação de uma placa em "L" no pilar zigomático. Figura 3-D: Teste de ducção forçada.

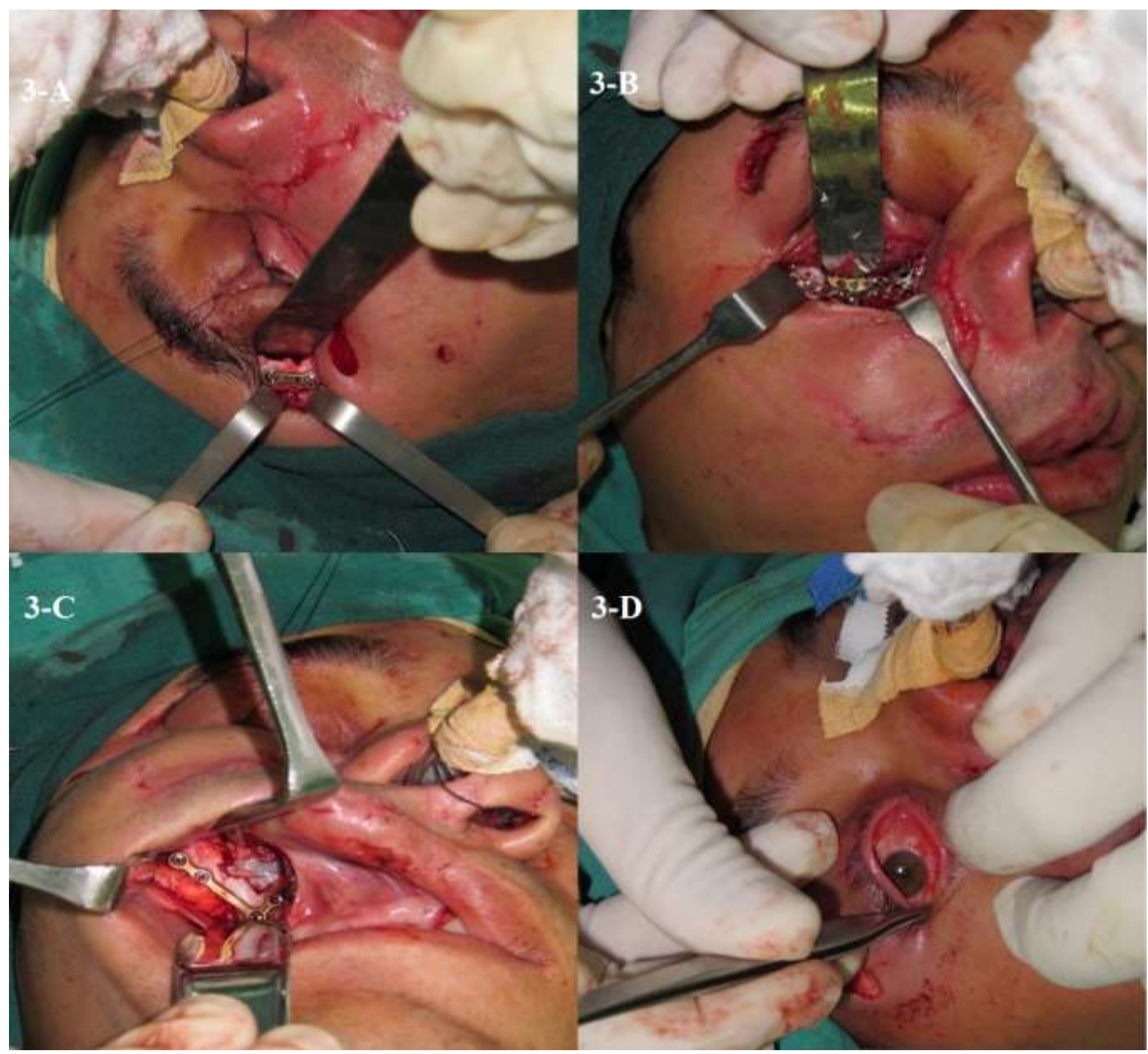

Fonte: Autores.

Foi realizado curativo de frost para impedir o surgimento de ectrópio, o mesmo consiste na sutura da conjuntiva bulbar e da terceira pálpebra para que a córnea esteja protegida e em contato com o tecido mole, o curativo foi deixado por 48 horas após a cirurgia. Após a cirurgia, o paciente teve a melhora na abertura de boca (Figura 4A), da equimose periorbitária, diplopia e oftalmoplegia no olho direito. Sendo assim, teve melhora da acuidade visual, e ganhos estéticos com a cicatrização de suas feridas pelo rosto. O paciente teve alta hospitalar após 3 dias do procedimento cirúrgico (Figura 4B).

Foi feito o acompanhamento do paciente pós cirúrgico com 1 mês, 75 dias e 11 meses (Figura 4C), realizando teste de movimentação dos olhos (Figura 4D e 4E), abertura bucal e solicitado um novo exame radiográfico (Figura 4F). 
Research, Society and Development, v. 10, n. 9, e17910917791, 2021

(CC BY 4.0) | ISSN 2525-3409 | DOI: http://dx.doi.org/10.33448/rsd-v10i9.17791

Figura 4-A: Abertura de boca do paciente pós cirurgia. Figura 4-B: 3 dias pós-operatório Figura 4-C: 11 meses pósoperatório Figura 4-D: Realização do teste ortóptico Figura 4-E: Realização do teste ortóptico. Figura 4-F: Tomografia pós cirúrgica.

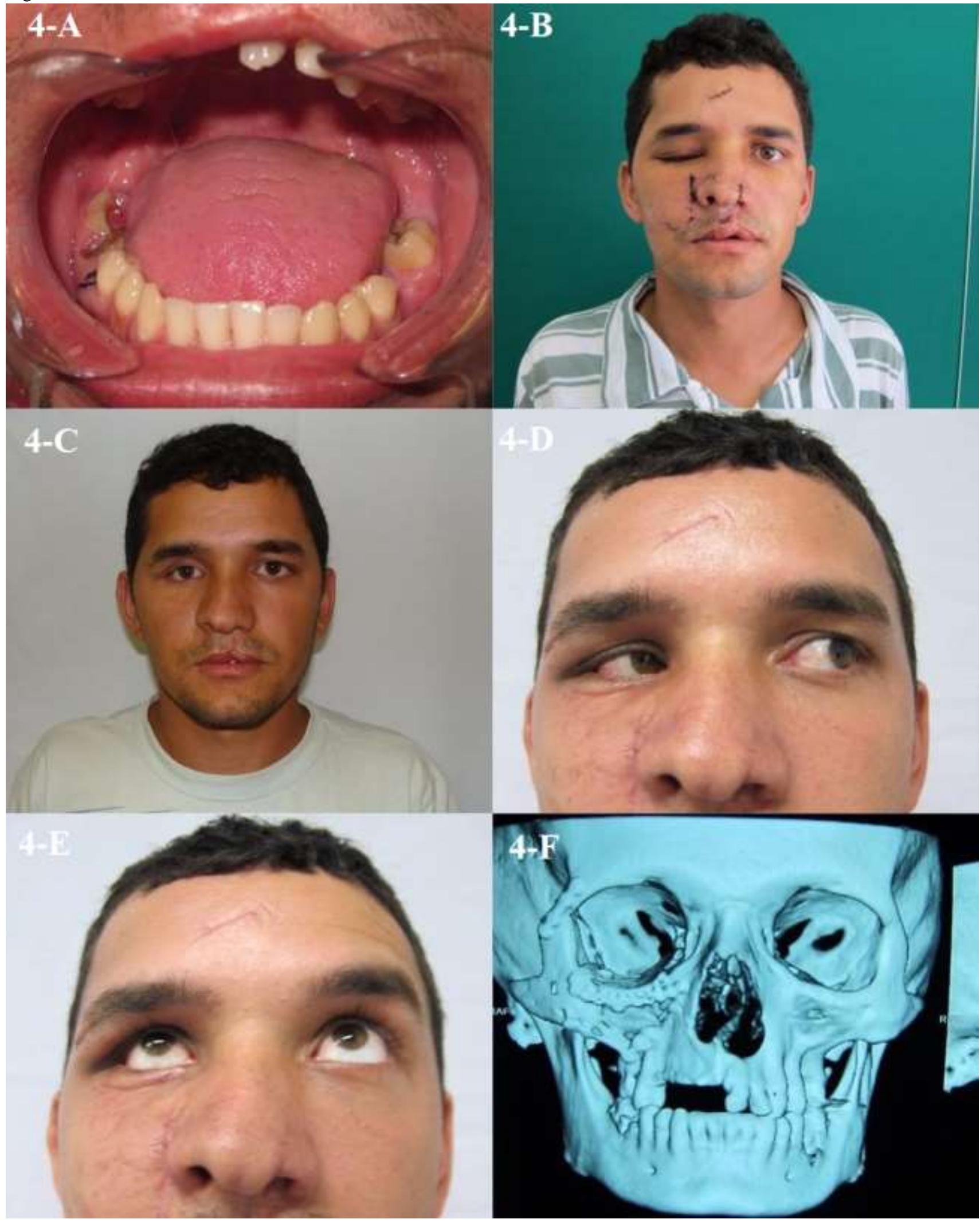

Fonte: Autores. 


\section{Resultados e Discussão}

Fraturas no terço médio da face são um grande desafio aos cirurgiões bucomaxilofaciais, pois essa região além de envolver tecido duro e mole, está também conectada a várias estruturas faciais, como o cérebro, olhos, seios da face, dentes e via aérea superior, tornando assim, uma situação que geralmente requer uma equipe multidisciplinar, podendo levar ao paciente diversas complicações estéticas e funcionais (Souza et al., 2018; Vasconcelos et al., 2014; Mendonça et al., 2016). No presente caso, o paciente apresentou como sinais e sintomas do trauma facial: ptose palpebral, equimose periorbitária, hiposfagma, diplopia e oftalmoplegia no olho direito, enoftalmia, perda de projeção ântero-posterior do zigomático, edema difuso em terço médio de face, alargamento de terço médio de face e limitação de abertura bucal.

Para diagnosticar as fraturas faciais deve-se fazer o exame clínico e imaginológico, tendo a tomografia computadorizada como padrão ouro pois permite uma visualização nos cortes sagital, coronal, axial e reconstrução tridimensional. Além do mais, a tomografia computadorizada define a extensão do trauma e o padrão da fratura com mais precisão, facilitando o tratamento precoce (Souza et al., 2018; Vasconcelos et al., 2014). Nesse estudo foi solicitado exame tomográfico, no qual foi observado a presença de solução de continuidade compatível com fratura blow-out e fraturas no complexo zigomático do lado direito, observando assim o local, tamanho e padrão das fraturas.

A intervenção em fraturas do CZO requer a análise do nível de deslocamento da fratura e os prováveis fragmentos da mesma, pois são condições relevantes para decidir se o tratamento será aberto ou fechado. Normalmente, é necessária a redução e fixação interna no tratamento aberto, sendo esta redução tempo-dependente e as vezes complicada de se conseguir devido a sua alta capacidade de produção de tecido cicatricial osteóide, especialmente nas fraturas cominutivas, por conta da complexidade do CZO, e se a cirurgia não for executada corretamente, a assimetria facial ficará evidente (Bao et al., 2019; Higgins et al., 2019). Fraturas que acometem o complexo zigomático e orbita, é possível uma apropriada redução e fixação em até 21 dias depois do trauma. Quando efetuado precocemente, o tratamento das fraturas zigomático-orbitárias aparenta mostrar consequências positivas (Hwang et al., 2011; Lu et al., 2012). Quanto à fixação interna, é a mais frequente, sendo estabelecida como o melhor tratamento para estas fraturas pois assegura estabilidade eficaz para a restauração óssea (Peretti et al., 2017; ElAnwar et al., 2017). No presente caso, optou-se pelo tratamento aberto, em razão das múltiplas fraturas e por estarem interferindo na função. A cirurgia foi feita 7 dias após o paciente ter dado entrada no hospital, devido à espera do parecer do oftalmologista e a melhora do edema para que o cirurgião bucomaxilofacial tenha a referência no momento da cirurgia.

Para se fazer a fixação interna, o acesso que mais se utiliza para a região de pilar zigomático é o vestibular maxilar, já para a verificação da sutura frontozigomática o acesso supra-orbitário seria o mais indicado; e a incisão transcutânea subciliar ou transconjuntival é empregada para ter acesso ao arco infraorbital e o assoalho de órbita. Ellis e Zide (2006), Freitas (2006) e Mendonça et al. (2016), citam que os acessos mais indicados para a estruturas afetadas, são: periorbitários, pré-auriculares e intrabucais. Já Dakir et al. (2015), demonstra em sua análise, os benefícios de uma abordagem cirúrgica intraoral para reparo de fraturas no complexo zigomáticofacial, sendo a mais coerente a inexistência de cicatriz na pele do paciente. No entanto, tal intervenção é limitada em circunstâncias que necessitam inclusive de uma restauração de assoalho de órbita. Ou seja, teoricamente, a severidade da fratura, a especificação, restauração da função e estética vão decidir o protocolo cirúrgico ideal (Wahdini et al., 2019; Dakir et al., 2015). Concordando com os autores, durante o planejamento cirúrgico do caso, foram optados por realizar o acesso supra-orbitário onde se verificou a fratura na sutura frontozigomática, o acesso vestibular maxilar para colocação de uma placa em "L" no pilar zigomático, fixando o mesmo e na tentativa de conseguir uma simetria mais próxima do padrão, também foi feito o acesso intraoral, no qual tal objetivo foi alcançado.

O tipo de fixação selecionado vai depender de algumas condições, como por exemplo a redução anatômica do complexo zigomático, estabilidade apropriada, e o posicionamento do osso (Laverick et al., 2008; Raimundo et al., 2008). A instalação de mini placas de titânio para a fixação interna são comumente utilizadas, devido a presença de osso compacto 
sólido na região frontozigomática, margem infraorbital e contraforte zigomático (Sassi et al., 2009). Esta fixação pode ser de um, dois ou três pontos, dependendo da estabilidade do zigoma para escolha da fixação (Laverick et al., 2008; Raimundo et al., 2008). O uso da tela de titânio para reparações de fraturas blow-out é considerado uma predileção pelos cirurgiões devido seus benefícios, tais quais: materiais finos, de simples contorno, prontamente consolidado, conservam a sua forma, e possui competência para neutralizar a quantidade quando apropriadamente aperfeiçoada, sem a possibilidade de reabsorção. Também podem prontamente transpassar amplas deformidades solicitando uma rigorosa estrutura, são perceptíveis na radiografia e são esterilizáveis. Um dos benefícios do titânio é gerar poucos artefatos na tomografia computadorizada do que outros materiais metálicos (Ellis et al., 2004; Potter et al., 2004). Por ser frequentemente utilizada e recomendada, além das 3 fixações internas, neste estudo também foram utilizadas placas de titânio na tentativa de conceder ao paciente um formato de rosto sem afundamento, o que foi alcançado e a instalação da tela de titânio no assoalho para reconstrução da orbita.

Após o correto diagnóstico e tratamento, fazer acompanhamento do paciente é de grande relevância para esses tipos de caso, devido às complicações e sequelas, as quais podem levar à déficits sensoriais e, em algumas ocasiões, até funcionais (Jardim et al., 2014). No presente caso, o paciente foi acompanhado por 11 meses, sendo feitos testes ortópticos ao longo dos meses e solicitando exame imaginológico, na tentativa de detectar alguma sequela pós operatória, mas até o momento não foram encontradas, apenas pontos positivos, como redução do edema, melhora na abertura de boca, da equimose periorbitária, da diplopia e da oftalmoplegia no olho direito.

Tendo em vista que a face é o local de expressão onde todos os sentimentos são transmitidos, a compreensão das peculiaridades do traumatismo facial é de suma importância, pois prejudica a vida do indivíduo definitivamente e, quando mal tratados, provocam sequelas, afastando a pessoa da sociedade, podendo causar também incapacidade de trabalho (Dingman et al, 2001). Mediante as escolhas no traçado do planejamento cirúrgico, foi alcançado o sucesso no tratamento do paciente com fraturas no CZO e fratura blow out, possibilitando a reabilitação do esqueleto facial do paciente, revertendo repercussões estéticas e funcionais causadas pelo trauma, através aptidão do cirurgião bucomaxilofacial na redução e fixação das fraturas.

\section{Considerações Finais}

Tendo em vista os aspectos observados no decorrer deste artigo, dito que quanto maior o tempo para realizar a cirurgia após o trauma grandes chances de levar ao aparecimento de infecções e má consolidações do caso, adiando o tratamento ou tornando necessário cirurgias mais complexas trazendo malefícios ao paciente. Observando as múltiplas fraturas e a fratura blow out, os cirurgiões bucomaxilofaciais decidiram que a melhor opção foi realizar o tratamento cirúrgico de fixação interna. A escolha de um procedimento cirúrgico aberto com três acessos cirúrgicos e três pontos de fixação interna, mostra-se como um procedimento seguro e confiável.

Avaliando o paciente com fratura do complexo zigomático orbitário e constatado que houve melhora dos ganhos estéticos e funcionais. Restauração da abertura bucal e motricidade ocular devido a melhora da oftalmoplegia, no planejamento cirúrgico foi optado por acessos que tinham como objetivo não deixar o paciente com cicatrizes visíveis conquistando um resultado mais estético. Sendo assim o cirurgião bucomaxilofacial é o único profissional capacitado para tratar este tipo de fratura, devido ao conhecimento e preparo desse profissional, sobre o terço médio da face.

\section{Referências}

Allareddy, V., Allareddy, V., \& Nalliah, R. P. (2011). Epidemiology of facial fracture injuries. Journal of Oral and Maxillofacial Surgery, 69(10), 2613-2618.

Bagheri, S. C., Bell, R. B., \& Khan, H. A. (2013). Terapias atuais em cirurgia bucomaxilofacial. In Terapias atuais em cirurgia bucomaxilofacial (pp. 11221122). 
Baird, C., Kernohan, G., \& Coates, V. (2004). Outcomes of advanced trauma life support training: questioning the role of observer. Accident and emergency nursing, 12(3), 131-135.

Bao, T., Yu, D., Luo, Q., Wang, H., Liu, J., \& Zhu, H. (2019). Quantitative assessment of symmetry recovery in navigation-assisted surgical reduction of zygomaticomaxillary complex fractures. Journal of Cranio-Maxillofacial Surgery, 47(2), 311-319.

Batstone, M. D., Monsour, F. N., Pattel, P., \& Lynham, A. (2007). The patterns of facial injury suffered by patients in road traffic accidents: a case controlled study. International Journal of Surgery, 5(4), 250-254.

Burm, J. S., Chung, C. H., \& Oh, S. J. (1999). Pure orbital blowout fracture: new concepts and importance of medial orbital blowout fracture. Plastic and reconstructive surgery, 103(7), 1839-1849.

Dakir, A., Muthumani, T., Prabu, N. P., Mohan, R., \& Maity, A. (2015). One point fixation of zygomatic tripod fractures in the zygomatic buttress through Keen's intraoral approach: A review of 30 cases. Journal of pharmacy \& bioallied sciences, 7(Suppl 1), S238.

Dingman, R. O., \& Natvig, P. (2001). Cirurgia das fraturas faciais. In Cirurgia das fraturas faciais (pp. 376-376).

El-Anwar, M. W., Elsheikh, E., Hussein, A. M., Tantawy, A. A., \& Abdelbaki, Y. M. (2017). Transconjunctival versus subciliary approach to the infraorbital margin for open reduction of zygomaticomaxillary complex fractures: a randomized feasibility study. Oral and maxillofacial surgery, $21(2), 187-192$.

Ellis, E., \& Messo, E. (2004). Use of nonresorbable alloplastic implants for internal orbital reconstruction. Journal of oral and maxillofacial surgery, 62(7), $873-881$.

Ellis III, E., \& Zide, M. F. (2006). Acessos cirúrgicos ao esqueleto facial. In Acessos cirúrgicos ao esqueleto facial (pp. 252-252).

Erdmann, D., Follmar, K. E., DeBruijn, M., Bruno, A. D., Jung, S. H., Edelman, D., \& Marcus, J. R. (2008). A retrospective analysis of facial fracture etiologies. Annals of plastic surgery, 60(4), 398-403.

Freire, E. (2001). Trauma: a doença dos séculos. In Trauma: a doença dos séculos (pp. 1456-1456).

Freitas, R. (2006). Tratado de Cirurgia Bucomaxilofacial, São Paulo, 1. Livraria Santos Editora Com. Imp.

Higgins, A., Hurrell, M., Harris, R., Findlay, G., David, M., \& Batstone, M. (2019). A study protocol for a randomised controlled trial evaluating the effects of intraoperative computed tomography on the outcomes of zygomatic fractures. Trials, 20(1), 1-10.

Hwang, K., \& Kim, D. H. (2011). Analysis of zygomatic fractures. Journal of Craniofacial Surgery, 22(4), 1416-1421.

Jardim, E. C. G., Lima, H. C., Pereira, T. T. M., Masocatto, D. C., Oliveira, M. M., \& de Mendonça, J. C. G. (2014). Tratamento de fratura complexa de terço médio de face associada a ferimento extenso. Archives Of Health Investigation, 3(3).

Laverick, S., Patel, N., \& Jones, D. C. (2008). Maxillofacial trauma and the role of alcohol. British journal of oral and maxillofacial surgery, $46(7)$, 542-546.

Lu, W., Zhou, H., Xiao, C., Shen, Q., Lin, M., \& Fan, X. (2012). Late correction of orbital-zygomatic-maxillary fractures combined with orbital wall fractures. Journal of Craniofacial Surgery, 23(6), 1672-1676.

Martins, R. H. G., Ribeiro, C. B. H., Fracalossi, T., \& Dias, N. H. (2013). A lei seca cumpriu sua meta em reduzir acidentes relacionados à ingestão excessiva de álcool? Revista do Colégio Brasileiro de Cirurgiões, 40, 438-442.

Mendonça, J. C. G., Gaetti-Jardim, E. C., dos Santos, M. A., Ximenes, W. L. A., dos Santos, C. M., de Quadros, D. C., \& Macena, J. A. (2016). Tratamento cirúrgico de fratura do complexo zigomático orbital: relato de caso. Archives Of Health Investigation, 5(5).

Miloro, M., Ghali, G. E., Larsen, P. E., Waite, P. D., \& Of, P. S. P. (2004). Oral and maxillofacial surgery.

Montovani, J. C., Campos, L. M. P. D., Gomes, M. A., Moraes, V. R. S. D., Ferreira, F. D., \& Nogueira, E. A. (2006). Etiologia e incidência das fraturas faciais em adultos e crianças: experiência em 513 casos. Revista brasileira de otorrinolaringologia, 72(2), $235-241$.

Oliveira, J. A. G. P. (2000). Traumatologia Bucomaxilofacial e Reabilitação Morfofuncional. Grupo Gen-Livraria Santos Editora.

Peretti, N., \& MacLeod, S. (2017). Zygomaticomaxillary complex fractures: diagnosis and treatment. Current opinion in otolaryngology \& head and neck surgery, 25(4), 314-319.

Perry, M., \& Morris, C. (2008). Advanced Trauma Life Support (ATLS) and facial trauma: can one size fit all? : Part 2: ATLS, maxillofacial injuries and airway management dilemmas. International journal of oral and maxillofacial surgery, 37(4), 309-320.

Potter, J. K., \& Ellis, E. (2004). Biomaterials for reconstruction of the internal orbit. Journal of oral and maxillofacial surgery, 62(10), $1280-1297$.

Prado, B., Falchet Sobral, P. C., \& Lobo Leandro, L. F. (2011). Fraturas do complexo zigomático por acidentes de trânsito na cidade de São Paulo. Rev. bras. cir. cabeça pescoço.

Raimundo, R. D., Guerra, L. A. P., Antunes, A. A., Carvalho, R. W. F., \& Santos, T. S. (2008). Mandible fractures: a retrospective analysis of 27 cases. Rev Cir Traumatol Buco-maxilo-fac, 8(1), 57-62.

Rampa, S., Wilson, F. A., Tak, H. J., Roy, S., Wani, R. J., Markiewicz, M. R., \& Allareddy, V. (2019). Patient characteristics and causes of facial fractures in the state of California. Journal of Oral and Maxillofacial Surgery, 77(9), 1855-1866.

Sassi, L. M., Dissenha, J. L., Bezeruska, C., Guebur, M. I., Hepp, V., Radaelli, R. L., \& Cervantes, O. (2009). Fraturas de zigomático: revisão de 50 casos. Rev. bras. cir. cabeça pescoço. 
Research, Society and Development, v. 10, n. 9, e17910917791, 2021

(CC BY 4.0) | ISSN 2525-3409 | DOI: http://dx.doi.org/10.33448/rsd-v10i9.17791

SouzaI, E. M. R., \& RochaII, R. S. (2009). Reconstrução orbitária com tela de titânio: relato de dois casos. TC, 8, 3.

Souza, D. F. M. D., Santili, C., Freitas, R. R. D., Akkari, M., \& Figueiredo, M. J. P. S. S. D. (2010). Epidemiologia das fraturas de face em crianças num pronto-socorro de uma metrópole tropical. Acta Ortopédica Brasileira, 18, 335-338.

Souza Rosa, F. C. L., Ferreira, P. H. S. G., Monteiro, N. G., Castro, T. A., Oliveira, D., Gonçalves, P. Z., \& Okamoto, R. (2017). Redução cirúrgica de fratura complexa em terço médio da face. Archives Of Health Investigation, 6.

Wahdini, S. I., Dachlan, I., Seswandhana, R., Hutagalung, M. R., Putri, I. L., \& Afandy, D. (2019). Neglected orbitozygomaticomaxillary fractures with complications: A case report. International journal of surgery case reports, 62, 35-39.

Vasconcelos, B. G., Silva, L. A. C., Silva Júnior, A. F., Mohn Neto, C. R., \& Pereira, C. M. (2014). Perfil epidemiológico dos pacientes com fraturas faciais atendidos em um hospital de Goiânia-Goiás. J Health Sci Inst, 32(3), 241-45. 